\title{
ORTHOTROPIC CONDUCTIVITY RECONSTRUCTION WITH VIRTUAL RESISTIVE NETWORK AND FARADAY'S LAW
}

\author{
MIN-GI LEE, MIN-SU KO, AND YONG-JUNG KIM
}

\begin{abstract}
We obtain the existence and the uniqueness at the same time in the reconstruction of orthotropic conductivity in two space dimensions by using two sets of internal current densities and boundary conductivity. The curl free equation of Faraday's law is taken instead of the elliptic equation in a divergence form which is typically used in electrical impedance tomography. A reconstruction method based on layered bricks type virtual resistive network (VRN) is developed to reconstruct orthotropic conductivity with up to $40 \%$ multiplicative noise.
\end{abstract}

\section{Contents}

1. Introduction 1

2. Well-posedness in orthotropic conductivity reconstruction 3

2.1. Preliminaries 3

2.2. Well-posedness 4

3. VRN for orthotropic conductivity reconstruction $\quad 8$

3.1. Mimetic discretization 8

3.2. Domains of dependence and influence 10

3.3. VRN of layered bricks $\quad 10$

4. Numerical simulations $\quad 12$

4.1. Target conductivity and adding noises 13

4.2. Rectangular cells versus layered bricks $\quad 14$

4.3. Regularization $\quad 15$

4.4. Numerical stability tests 16

$\begin{array}{ll}\text { References } & 17\end{array}$

\section{INTRODUCTION}

Suppose that $\Omega \subset \boldsymbol{R}^{2}$ is a bounded convex domain of an electrical conductivity body with a smooth boundary $\partial \Omega$ and $\mathbf{F}_{k}: \Omega \rightarrow \boldsymbol{R}^{2}, k=1,2$, are given (electrical current density) vector fields. The first purpose of this paper is to show the well-posedness in finding an orthotropic resistivity distribution

$$
\boldsymbol{r}(\mathbf{x}):=\left(\begin{array}{cc}
r^{11}(\mathbf{x}) & 0 \\
0 & r^{22}(\mathbf{x})
\end{array}\right), \quad \mathbf{x}:=(x, y) \in \Omega \subset \boldsymbol{R}^{2}
$$

that satisfies

$$
\begin{aligned}
& \nabla \times\left(\boldsymbol{r} \mathbf{F}_{k}\right)=0 \text { in } \Omega, k=1,2, \\
& r^{i i}=b^{i} \text { on } \Gamma_{i}^{-} \subset \partial \Omega, i=1,2 .
\end{aligned}
$$

Date: January 6, 2015.

Key words and phrases. electrical impedance tomography, current density imaging, MREIT, virtual resistive network. 
Here, $b^{i}(\mathbf{x})$ is a boundary value given on a portion of boundary $\Gamma_{i}^{-} \subset \partial \Omega$. The inverse of the resistivity tensor, $\boldsymbol{\sigma}=\boldsymbol{r}^{-1}$, is called the conductivity tensor. The second purpose is to develop a virtual resistive network (or VRN for brevity) method to construct the orthotropic resistivity numerically.

The curl free equation in (1.2) is the third Maxwell's equation, or Faraday's law, for the static electromagnetism. This system gives a direct relation between the unknown resistivity tensor $\boldsymbol{r}$ and the given vector field $\mathbf{F}$, which makes the analysis and computation direct. The electrical current density is sometimes denoted by the letter $\mathbf{J}$ and we reserve the letter for the case without a noise. However, a noise is included for various reasons and we denote a noised current density by the letter $\mathbf{F}$. The existence of a resistivity tensor $\boldsymbol{r}$ that satisfies (1.2) is obvious if $\mathbf{F}_{1}$ and $\mathbf{F}_{2}$ are exact current densities generated by an existing orthotropic conductivity body. However, if a noise is included in these vector fields, the existence is not guaranteed. Therefore, we start our study by classifying admissible vector fields $\mathbf{F}_{k}$ 's that allow the existence (see Definition 2.2). It is important not to make the problem over determined to obtain existence and hence the right amount of boundary value should be specified, where such portion $\Gamma_{i}^{-}$'s are given in Lemma 2.4. The well-posedness of the problem is finally obtained in Theorem 2.5 using the Picard type iteration method. This contraction mapping method also gives the uniqueness and the stability naturally.

In Section 3 a VRN method is developed for the orthotropic resistivity. If $r^{11}=r^{22}$ on $\Omega$, the conductivity is called isotropic. However, for a conductivity body with a muscle fiber structure, anisotropic model is used which is given by a $2 \times 2$ symmetric matrix. In this context an orthotropic conductivity models a local behavior of a muscle fiber structure when eigenvectors are aligned in the directions of $x$ and $y$ axis. Hence the orthotropic conductivity is an intermediate stage that connects isotropic and anisotropic conductivities. However, the orthotropic case gives an extra difficulty in reconstructing the conductivity. Since the domains of dependence are the horizontal and vertical lines, one may observe noise stripes as in Figure $9(\mathrm{a}, \mathrm{d})$. This is an extreme case of the stripes observed from isotropic cases (see [17, Figures 12-20]). To reduce such a symptom a layered bricks type of VRN is introduced (see Figures 4 and 5). This type of VRN successfully reduces the noise stripes (see Figures 9(b,f)). Numerical simulation results are given in Section 4.

The motivation of the problem is the current density impedance imaging (CDII for brevity) or a magnetic resonance imaging based electrical impedance tomography (MREIT) problem. The conductivity tensor $\boldsymbol{\sigma}=\boldsymbol{r}^{-1}$ and the electrical potential $u$ satisfy

$$
\begin{array}{ll}
\nabla \cdot(\boldsymbol{\sigma} \nabla u)=0 & \text { in } \Omega, \\
-\boldsymbol{\sigma} \nabla u \cdot \mathbf{n}=g & \text { on } \partial \Omega,
\end{array}
$$

where $\mathbf{n}$ is the outward unit normal vector to the boundary $\partial \Omega$ and $g$ is the normal component of the boundary current that satisfies $\int_{\partial \Omega} g d s=0$. This elliptic equation connects the Neumann current data $\left.g\right|_{\partial \Omega}$ to the Dirichlet potential data $\left.u\right|_{\partial \Omega}$. The electrical impedance tomography (or EIT) is the study of this relation (see $[3,4,5,8,22,25,26]$ and references therein) and, in particular, it is known that only an isotropic conductivity is reconstructed uniquely from this relation with all possible boundary observations. Another option is using internal measurements. For example, MRI technology allows us to measure the current density $\mathbf{J}$ in the domain $\Omega$. Then, the voltage $u$ satisfy Ohm's law:

$$
\mathbf{J}=-\boldsymbol{\sigma} \nabla u \quad \text { in } \Omega .
$$

The uniqueness of isotropic conductivity with such an internal measurement has been studied by several authors (see $[10,11,12,13,14,20,21]$ ). The uniqueness in anisotropic conductivity reconstruction has been studied recently (see $[6,7,9,18,19]$ ). 
The main difference of the study in this paper is the choice of Faraday's law (1.2) instead of the system (1.3)-(1.4). This choice simplifies the problem and allows us to obtain the existence together with the uniqueness by providing the right amount of current and boundary data. Numerically, VRN is constructed instead of FDM, which is a result of loop integrals for the curl free equation. For the isotropic case, $r^{11}=r^{22}$, the corresponding VRN method and the well-posedness of the problem has been studied in $[16,17]$ using a single vector field, i.e., $k=1$. Remember that, for the orthotropic case of this paper, two sets of internal current densities, $k=2$, are needed. For a fully anisotropic case, Bal et al. [6, 7] obtained the uniqueness of anisotropic conductivity when four sets of current densities are given. Using a similar method of this paper based on Faraday's law, it has been shown in [15] that three sets of current densities, $k=3$, are needed to obtain the existence and uniqueness for the anisotropic conductivity reconstruction.

\section{WELL-POSEDNESS IN ORTHOTROPIC CONDUCTIVITY RECONSTRUCTION}

2.1. Preliminaries. In this section, we introduce notations and review fundamental relations among current density $\mathbf{J}$, electrical field $\mathbf{E}$, voltage $u$, stream function $\psi$, conductivity $\boldsymbol{\sigma}$ and resistivity $\boldsymbol{r}$. These are basic relations in static electromagnetism and will be used in the following sections.

Let $\Omega \subset \boldsymbol{R}^{2}$ be simply connected bounded domain with smooth boundary and $\mathbf{J}$ and $\mathbf{E}$ be smooth on $\Omega$. The current density $\mathbf{J}$ and the electrical field $\mathbf{E}$ are vector fields which are incompressible and irrotational, respectively, i.e.,

$$
\nabla \cdot \mathbf{J}=0 \text { and } \nabla \times \mathbf{E}=0 .
$$

Therefore, there exist a stream function $\psi$ and a potential $u$ such that

$$
\mathbf{J}=\nabla^{\perp} \psi \quad \text { and } \quad \mathbf{E}=-\nabla u, \quad \text { where } \nabla^{\perp}:=\left(\begin{array}{c}
\partial_{y} \\
-\partial_{x}
\end{array}\right)
$$

The resistivity $\boldsymbol{r}$ and the conductivity $\boldsymbol{\sigma}$ are $2 \times 2$ positive symmetric matrixes with $\boldsymbol{\sigma}=\boldsymbol{r}^{-1}$ and Ohm's Law is written as

$$
\mathbf{J}=\sigma \mathbf{E} \quad \text { or } \quad \mathbf{E}=\boldsymbol{r} \mathbf{J} .
$$

The substitution of Ohm's law into (2.1) gives

$$
\nabla \cdot(\boldsymbol{\sigma} \mathbf{E})=0 \text { and } \nabla \times(\boldsymbol{r} \mathbf{J})=0 .
$$

If the electrical field $\mathbf{E}$ is given, the first equation is useful to find the conductivity $\boldsymbol{\sigma}$ (see $[1,23,24])$. If the current density $\mathbf{J}$ is given, the second equation gives a direct relation for the resistivity tensor $\boldsymbol{r}$.

Suppose that $\boldsymbol{\sigma}$ or $\boldsymbol{r}$ is given and we want to find $\mathbf{E}$ or $\mathbf{J}$. It is clear that these scalar equations are inconvenient to find the vector fields $\mathbf{E}$ or $\mathbf{J}$. In fact, equations for voltage $u$ or the stream function $\psi$ are usually used. If (2.2) and (2.3) are substituted into (2.1), one obtains

$$
\nabla \cdot(\boldsymbol{\sigma} \nabla u)=0 \text { and } \nabla \times\left(\boldsymbol{r} \nabla^{\perp} \psi\right)=0 .
$$

A typical example for the potential $u$ is a Neumann problem given by

$$
\begin{array}{ll}
\nabla \cdot(\boldsymbol{\sigma} \nabla u)=0, & \text { in } \Omega, \\
-\boldsymbol{\sigma} \nabla u \cdot \mathbf{n}=g, & \text { on } \partial \Omega,
\end{array}
$$

where the boundary data satisfies $\oint_{\partial \Omega} g d s=0$. The choice of boundary condition depends on each situation and one may also take Dirichlet boundary condition, too. Similarly, one may obtain an equation for the stream function $\psi$ from (2.5). However, we may also write the 
equation in a divergence form and use the theories developed for it. The corresponding equation is

$$
\begin{aligned}
\nabla \cdot(\tilde{\boldsymbol{r}} \nabla \psi)=0, & \text { in } \Omega, \\
\psi=G, & \text { on } \partial \Omega,
\end{aligned}
$$

where $\tilde{\boldsymbol{r}}$ is a positive definite matrix,

$$
\tilde{\boldsymbol{r}}=\left(\begin{array}{rr}
r^{22} & -r^{21} \\
-r^{12} & r^{11}
\end{array}\right)
$$

and a Dirichlet boundary condition is imposed. The two problems are connected through the boundary values. Note that

$$
g=-\boldsymbol{\sigma} \nabla u \cdot \mathbf{n}=\left(\partial_{y} \psi,-\partial_{x} \psi\right) \cdot\left(n^{1}, n^{2}\right)=\nabla \psi \cdot\left(-n^{2}, n^{1}\right),
$$

where $\left(-n^{2}, n^{1}\right)$ is the unit tangent vector of $\partial \Omega$ that rotates the boundary counterclockwise. Let $L$ be the arc-length of the boundary $\partial \Omega$ and $\gamma(s):[0, L] \rightarrow \partial \Omega$ be the parameterized curve rotating the boundary counterclockwise with unit speed $\left|\gamma^{\prime}(s)\right|=1$. Then

$$
\frac{d}{d s} G(\gamma(s))=\gamma^{\prime}(s) \cdot \nabla G(\gamma(s))=\left(-n^{2}, n^{1}\right) \cdot \nabla \psi(\gamma(s))=g(\gamma(s)) .
$$

Now we introduce a lemma which will be used in the proof of existence of admissibility of current density.

Lemma 2.1 (Alessandrini [2]). Let $\Omega \subset \boldsymbol{R}^{2}$ be a bounded simply connected domain, $a^{i j} \in C^{1}(\Omega)$, $b^{i} \in C(\Omega), i, j=1,2$, and $G \in C(\bar{\Omega})$. Suppose that $u \in W_{\text {loc }}^{2}(\Omega) \cap C(\bar{\Omega})$ satisfies

$$
\sum_{i, j=1}^{2} a^{i j} \partial_{x_{i}} \partial_{x_{j}} u+\sum_{i=1}^{2} b^{i} \partial_{x_{i}} u=0 \text { in } \Omega \text { and } u=G \text { on } \partial \Omega \text {. }
$$

If $\left.G\right|_{\partial \Omega}$ has $N$ number of maxima on $\partial \Omega$, then the number of interior critical points of $u$ are less than $N-1$ including their multiplicities.

2.2. Well-posedness. In this section we show the well-posedness of the the inverse problem of finding the resistivity $\boldsymbol{r}$ that satisfies (1.2). It is clear that the existence fails if the given data $\mathbf{F}_{1}$ and $\mathbf{F}_{2}$ are arbitrary. First, we define the space for the vector fields that gives the the existence of such resistivity. Let $\Omega \subset \boldsymbol{R}^{2}$ be a bounded convex domain with a smooth boundary.

Definition 2.2. Let $\Omega \subset \boldsymbol{R}^{2}$ be a simply connected bounded domain. Two sets of smooth vector fields $\mathbf{F}_{1}, \mathbf{F}_{2}: \Omega \rightarrow \boldsymbol{R}^{2}$ are called admissible pair if $\mathbf{F}_{1} \times \mathbf{F}_{2} \neq 0$ for all $\mathbf{x} \in \bar{\Omega}$.

The following theory is useless if there is not any admissible pair or one cannot generate such admissible current densities for a given domain $\Omega$ and a conductivity tensor $\boldsymbol{\sigma}$. Hence we first show how admissible pair of current densities can be obtained.

Proposition 2.3. Let $\Omega$ be a bounded simply connected domain and $\boldsymbol{r}$ be a symmetric positive definite matrix field defined on $\bar{\Omega}$. Suppose that the perimeter of the boundary $\partial \Omega$ is $L>0$ and let $\gamma:[0, L] \rightarrow \partial \Omega$ be the parameterized curve rotating the boundary counterclockwise with unit speed $\left|\gamma^{\prime}(s)\right|=1$. Let $G_{k}: \partial \Omega \rightarrow \boldsymbol{R}$ be given by $G_{1}(\gamma(s))=-\cos \left(\frac{2 \pi}{L} s\right)$ and $G_{2}(\gamma(s))=\sin \left(\frac{2 \pi}{L} s\right)$. Let $\psi_{k}$ be the solution of

$$
\begin{aligned}
\nabla \cdot\left(\boldsymbol{r} \nabla \psi_{k}\right) & =0, \quad \text { in } \Omega, \\
\psi & =G_{k}, \quad \text { on } \partial \Omega .
\end{aligned}
$$

Then, $\nabla^{\perp} \psi_{1}(\mathbf{x}) \times \nabla^{\perp} \psi_{2}(\mathbf{x}) \neq 0$ for all $\mathbf{x} \in \Omega$. 
Proof. For both of $G_{1}$ and $G_{2}$, there is only one local maximum along the boundary. Since the boundary is assumed to be sufficiently smooth, one can extend $G_{k}, k=1,2$ into the $\bar{\Omega}$ smoothly. It is clear that only the local maxima along the boundary can be local maxima of extended functions along the boundary. By Alessandrini 2.1, $\nabla \psi_{1}$ and $\nabla \psi_{2}$ are non-vanishing in $\Omega$. By Hopf's lemma, $\nabla \psi_{k} \neq 0$ along the boundary. Suppose that there is a point $\mathbf{x}_{0} \in \Omega$ such that $\nabla \psi_{1}\left(\mathbf{x}_{0}\right) \times \nabla \psi_{2}\left(\mathbf{x}_{0}\right)=0$. Then, $\nabla \psi_{1}\left(\mathbf{x}_{\mathbf{0}}\right)=c \nabla \psi_{2}\left(\mathbf{x}_{\mathbf{0}}\right)$ for some constant $c$. Then, $\tilde{\psi}=\psi_{1}-c \psi_{2}$ is also a solution with a boundary condition $\tilde{\psi}(\gamma(s))=-\cos \left(\frac{2 \pi}{L} s\right)-c \sin \left(\frac{2 \pi}{L} s\right)=$ $-\sqrt{\left(1+c^{2}\right)} \sin \left(\frac{2 \pi}{L}\left(s+s_{*}\right)\right)$ for some $s_{*}$ and $\mathbf{x}_{0}$ is an interior critical point. However, this boundary condition also has one maximum and hence $\tilde{\psi}$ cannot have a critical point, which is a contradiction. Therefore $\nabla \psi_{1} \times \nabla \psi_{2}$ has no interior zero point.

This proposition gives a method to find admissible current density pair for any bounded simply connected domain with smooth boundary by simply taking $\mathbf{F}_{k}=\nabla^{\perp} \psi_{k}$. One may choose the boundary condition $G_{k}$ rather freely as long as it has a unique local maximum or a single oscillation. One may also obtain the current density by solving (2.6) with boundary values $g_{k}(\gamma(s))=\frac{d}{d s} G_{k}(\gamma(s))$ which is from the relation (2.8). Then, the current densities are given by $\mathbf{F}_{k}=-\boldsymbol{\sigma} \nabla u_{k}$.

The uniqueness and the existence of the resistivity that satisfies (1.2) depend on the correct choice of boundaries $\Gamma_{i}$. The convexity assumption for the domain is involved from this part for a simpler proof and presentation.

Lemma 2.4. Let $\Omega \subset \boldsymbol{R}^{2}$ be a bounded convex domain with a smooth boundary and $\left(n^{1}(\mathbf{x}), n^{2}(\mathbf{x})\right)$ be the outward unit normal vector to the boundary. Divide the boundary into three parts,

$$
\begin{aligned}
& \Gamma_{1}^{+}:=\left\{\mathbf{x} \in \partial \Omega \mid n^{2}(\mathbf{x})>0\right\}, \\
& \Gamma_{1}^{-}:=\left\{\mathbf{x} \in \partial \Omega \mid n^{2}(\mathbf{x})<0\right\}, \\
& \Gamma_{1}^{0}:=\left\{\mathbf{x} \in \partial \Omega \mid n^{2}(\mathbf{x})=0\right\} .
\end{aligned}
$$

Then, $\Gamma_{1}^{+}$and $\Gamma_{1}^{-}$are connected and $\Gamma_{1}^{0}$ consists of two connected components that connect $\Gamma_{1}^{+}$ and $\Gamma_{1}^{-}$(see Figure 1(a)). Similarly, we may divide the boundary into three parts as

$$
\begin{aligned}
& \Gamma_{2}^{+}:=\left\{\mathbf{x} \in \partial \Omega \mid n^{1}(\mathbf{x})>0\right\} \\
& \Gamma_{2}^{-}:=\left\{\mathbf{x} \in \partial \Omega \mid n^{1}(\mathbf{x})<0\right\}, \\
& \Gamma_{2}^{0}:=\left\{\mathbf{x} \in \partial \Omega \mid n^{1}(\mathbf{x})=0\right\},
\end{aligned}
$$

where $\Gamma_{2}^{ \pm}$are connected and $\Gamma_{2}^{0}$ consists of two components that connect $\Gamma_{2}^{+}$and $\Gamma_{2}^{-}$(see Figure $1(b))$.

The proof of the lemma is trivial since the domain is convex and is omitted. Instead an illustration is given in Figure 1. The boundary $\Gamma_{1}^{-}$and $\Gamma_{1}^{0}$ are denoted in Figure 1(a). One may find that $\Gamma_{1}^{0}$ has two components on the left and the right sides of the figure, which are veritcal parts. If the domain is a circle, it consists of two points. The bottom side of the boundary is $\Gamma_{1}^{-}$as denoted in the figure. The part without any mark is $\Gamma_{1}^{+}$. In Figure 1(b) the boundary $\Gamma_{2}^{-}$and $\Gamma_{2}^{0}$ are denoted. Boundary $\Gamma_{2}^{0}$ consists of two horozontal components on the top and the bottom. Now the left side boundary is $\Gamma_{2}^{-}$as denoted in the figure. The other part without a mark is $\Gamma_{2}^{+}$.

The system of two sets of Faraday's law for the orthotropic problem are written as

$$
\begin{aligned}
& \nabla \times\left(\boldsymbol{r} \mathbf{F}_{1}\right)=0, \\
& \nabla \times\left(\boldsymbol{r} \mathbf{F}_{2}\right)=0,
\end{aligned}
$$




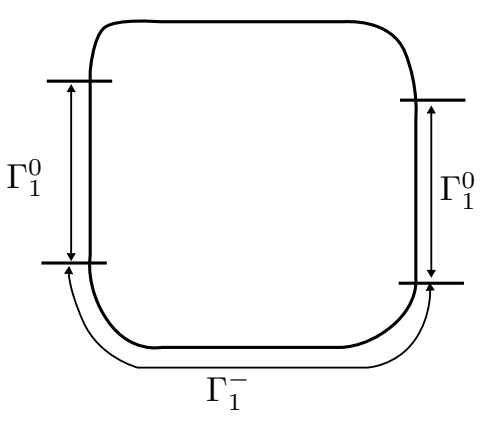

(a) $\Gamma_{1}^{-}$and $\Gamma_{1}^{0}$

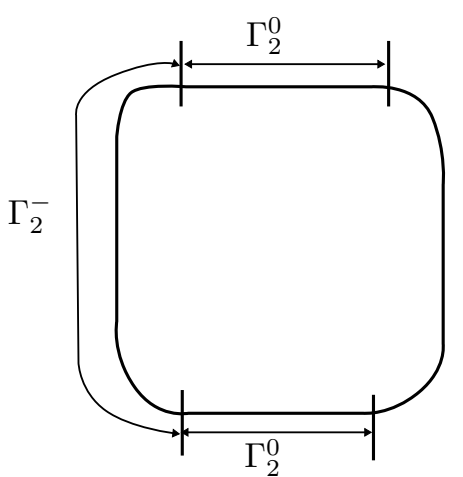

(b) $\Gamma_{2}^{-}$and $\Gamma_{2}^{0}$

Figure 1. The geometry of boundary

where $\boldsymbol{r}=r^{i j} \delta^{i j}$ is a diagonal matrix field. The system is written as

$$
\left(\begin{array}{c}
\partial_{y} r^{11} \\
\partial_{x} r^{22}
\end{array}\right)=\left(\begin{array}{ll}
-F_{1}^{x} & F_{1}^{y} \\
-F_{2}^{x} & F_{2}^{y}
\end{array}\right)^{-1}\left(\begin{array}{ll}
\partial_{y} F_{1}^{x} & -\partial_{x} F_{1}^{y} \\
\partial_{y} F_{2}^{x} & -\partial_{x} F_{2}^{y}
\end{array}\right)\left(\begin{array}{c}
r^{11} \\
r^{22}
\end{array}\right)=:\left(\begin{array}{l}
a^{11} r^{11}+a^{12} r^{22} \\
a^{21} r^{11}+a^{22} r^{22}
\end{array}\right)
$$

Notice that the inverse matrix in the equation exists if $\mathbf{F}_{1} \times \mathbf{F}_{2} \neq 0$ and the coefficients $a^{i j}$ are continuous if $\mathbf{F}_{1}$ and $\mathbf{F}_{2}$ are smooth. The characteristic lines for the eigenvalues $r^{11}$ and $r^{22}$ are vertical and horizontal lines, respectively. Therefore, one should avoid boundary values along characteristic lines for the existence of a solutions which is the reason why we divided the boundary as in Lemma 2.4 .

Theorem 2.5. Let $\Omega$ be a convex domain with a smooth boundary, $\mathbf{F}_{1}, \mathbf{F}_{2}: \Omega \rightarrow \boldsymbol{R}^{2}$ be a smooth admissible pair, and $b^{i}: \Gamma_{i}^{-} \rightarrow \boldsymbol{R}^{+}, i=1,2$, are bounded away from zero, where $\Gamma_{i}^{-} \subset \partial \Omega$ are given by (2.9) and (2.10). Then, there exists a unique orthotropic resistivity $\boldsymbol{r}=\left(r^{i j} \delta^{i j}\right)$ with

$$
r^{11}, r^{22} \in C(\bar{\Omega})
$$

such that $r^{11}$ and $r^{22}$ are differentiable with respect to $y$ and $x$ variables, respectively, and satisfy

$$
\begin{array}{ll}
\nabla \times\left(\boldsymbol{r} \mathbf{F}_{k}\right)=0 & \text { in } \Omega, k=1,2, \\
r^{i i}(\mathbf{x})=b^{i}(\mathbf{x}) & \text { on } \Gamma_{i}^{-} \subset \partial \Omega, i=1,2 .
\end{array}
$$

Let $\tilde{\boldsymbol{r}}=\left(\tilde{r}^{i j} \delta^{i j}\right)$ be another solution with admissible current density fields $\tilde{\mathbf{F}}_{k}$ and boundary data $\tilde{b}^{1}$ and $\tilde{b}^{2}$. Then, there exists a constant $C=C\left(\Omega, \mathbf{F}_{k}, \nabla \mathbf{F}_{k}, \tilde{\mathbf{F}}_{k}, \nabla \tilde{\mathbf{F}}_{k}, b^{i}, \tilde{b}^{i}\right)$ such that

$$
\|\boldsymbol{r}-\tilde{\boldsymbol{r}}\|_{L^{\infty}(\Omega)} \leq C\left(\sum_{k=1}^{2}\left\|\mathbf{F}_{k}-\tilde{\mathbf{F}}_{k}\right\|_{C^{1}(\Omega)}+\left\|b^{1}-\tilde{b}^{1}\right\|_{L^{\infty}\left(\Gamma_{1}^{-}\right)}+\left\|b^{2}-\tilde{b}^{2}\right\|_{L^{\infty}\left(\Gamma_{2}^{-}\right)}\right) .
$$

Proof. The existence part of the proof is based on a Picard type iteration method. First, we rewrite the differential equations in (2.11) into integral equations. For a given pair $f^{1}, f^{2}: \bar{\Omega} \rightarrow$ $\boldsymbol{R}$, define

$$
\begin{gathered}
k^{1}\left(f^{1}, f^{2}\right)(x, y)=b^{1}\left(x, y_{0}(x)\right)+\int_{y_{0}(x)}^{y} a^{11} f^{1}(x, t)+a^{12} f^{2}(x, t) d t, \\
k^{2}\left(f^{1}, f^{2}\right)(x, y)=b^{2}\left(x_{0}(y), y\right)+\int_{x_{0}(y)}^{x} a^{21} f^{1}(t, y)+a^{22} f^{2}(t, y) d t,
\end{gathered}
$$

where $\left(x, y_{0}(x)\right) \in \Gamma_{1}^{-}$and $\left(x_{0}(y), y\right) \in \Gamma_{2}^{-}$are the boundary points that intersect vertical and horizontal lines, respectively, that pass the point $(x, y)$. The uniqueness of such points is clear from the convexity of $\Omega$. We define an operator $K:=\left(k^{1}, k^{2}\right)$. 


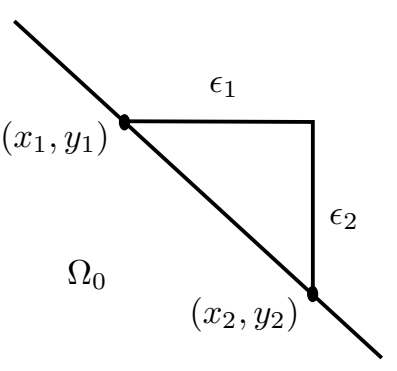

(a) non-characteristic boundary of $\Omega_{0}$

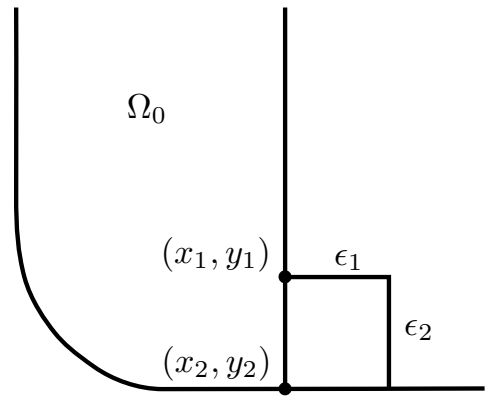

(b) characteristic boundary of $\Omega_{0}$

FiguRE 2. Illustrations for the proof of Theorem 2.5.

Let $r_{0}^{1}=r_{0}^{2}=1$ on $\bar{\Omega}$ and define a sequence as $\left(r_{n+1}^{1}, r_{n+1}^{2}\right):=K\left(r_{n}^{1}, r_{n}^{2}\right)$ for $n \geq 0$. First we show that $\left(r_{n}^{1}, r_{n}^{2}\right)$ converges on $\bar{\Omega}$ as $n \rightarrow \infty$. Let

$$
A=\left\{(x, y) \in \bar{\Omega}:\left(r_{n}^{1}(x, y), r_{n}^{2}(x, y)\right) \text { converges as } n \rightarrow \infty\right\} .
$$

Since $r_{n}^{i}(x, y)=b^{i}(x, y)$ for all $(x, y) \in \Gamma_{1}^{-} \cap \Gamma_{2}^{-}$and $n \geq 1, \Gamma_{1}^{-} \cap \Gamma_{2}^{-} \subset A$. Let $\Omega_{0}$ be the connected component of $A$ that includes $\Gamma_{1}^{-} \cap \Gamma_{2}^{-}$. We will show that $\Omega_{0}=\bar{\Omega}$. We will suppose that $\Omega_{0} \neq \bar{\Omega}$ and derive a contradiction. There are two possible cases. First, consider the case that there exists a point $\left(x_{1}, y_{1}\right) \in \partial \Omega_{0} \cap \Omega$ such that the boundary is not vertical nor horizontal at the point $\left(x_{1}, y_{1}\right)$ (see Figure $\left.2(\mathrm{a})\right)$. Let $(x, y)$ be a point in the triangular shape region of the figure. Then,

$$
\begin{aligned}
k^{1}\left(r_{n+1}^{1}, r_{n+1}^{2}\right)-k^{1}\left(r_{n}^{1}, r_{n}^{2}\right)= & \int_{\tilde{y}_{0}(x)}^{y} a^{11}\left(r_{n+1}^{1}-r_{n}^{1}\right)(x, t)+a^{12}\left(r_{n}^{2}-r_{n}^{2}\right)(x, t) d t \\
& +\int_{y_{0}(x)}^{\tilde{y}_{0}(x)} a^{11}\left(r_{n+1}^{1}-r_{n}^{1}\right)(x, t)+a^{12}\left(r_{n}^{2}-r_{n}^{2}\right)(x, t) d t
\end{aligned}
$$

where $\left(x, \tilde{y}_{0}(x)\right)$ is the intersection point between the boundary $\partial \Omega_{0}$ and the vertical line that passes $(x, y)$. The second integral converges to zero as $n \rightarrow \infty$ since $\left(x, \tilde{y}_{0}(x)\right) \in \Omega_{0}$. The first integral is estimated by

$$
\int_{y_{0}(x)}^{\tilde{y}_{0}(x)} a^{11}\left(r_{n+1}^{1}-r_{n}^{1}\right)(x, t)+a^{12}\left(r_{n}^{2}-r_{n}^{2}\right)(x, t) d t \leq \epsilon_{2}\left(\max _{i, j=1,2}\left|a^{i j}\right|\right)\left\|\left(r_{n+1}^{1}, r_{n+1}^{2}\right)-\left(r_{n}^{1}, r_{n}^{2}\right)\right\|_{\infty},
$$

where the uniform norm is taken on the triangular domain. We may obtain a similar estimate for " $k^{2}\left(r_{n+1}^{1}, r_{n+1}^{2}\right)-k^{2}\left(r_{n}^{1}, r_{n}^{2}\right)$ " and conclude that

$$
\left\|K\left(r_{n+1}^{1}, r_{n+1}^{2}\right)-K\left(r_{n}^{1}, r_{n}^{2}\right)\right\|_{\infty} \leq\left(\epsilon_{1}+\epsilon_{2}\right)\left(\max _{i, j=1,2}\left|a^{i j}\right|\right)\left\|\left(r_{n+1}^{1}, r_{n+1}^{2}\right)-\left(r_{n}^{1}, r_{n}^{2}\right)\right\|_{\infty}+o(n)
$$

as $n \rightarrow \infty$. Therefore, if $\epsilon_{1}+\epsilon_{2}<\frac{1}{\max _{i, j=1,2}\left|a^{i j}\right|}$, then $K$ is an contraction mapping on the domain and hence we may conclude that $\Omega_{0}$ is not a connected component of the set $A$ if $\Omega_{0} \neq \bar{\Omega}$ since it can be expanded. Now suppose that the boundary $\partial \Omega_{0} \cap \Omega$ consists of vertical or horizontal lines only (see Figure 2(b) for a vertical line case). Then, we may apply the previous arguments on a small region where conductivity on the bottom and left side boundaries are previously obtained. Therefore, we conclude that $r_{n}^{1}(x, y)$ and $r_{n}^{2}(x, y)$ converge as $n \rightarrow \infty$ for all $(x, y) \in \bar{\Omega}$.

Let $r_{n}^{i} \rightarrow r^{i i}$ for $i=1,2$. Then, the limits satisfy the integral equations

$$
\begin{aligned}
& r^{11}(x, y)=b^{1}\left(x, y_{0}(x)\right)+\int_{y_{0}(x)}^{y}\left(a^{11} r^{11}(x, t)+a^{12} r^{22}(x, t)\right) d t, \\
& r^{22}(x, y)=b^{2}\left(x_{0}(y), y\right)+\int_{x_{0}(y)}^{x}\left(a^{21} r^{11}(t, y)+a^{22} r^{22}(t, y)\right) d t .
\end{aligned}
$$


Clearly, $r^{11}$ is differentiable with respect to the $y$ variable and $r^{22}$ with respect to the $x$ variable and satisfy the boundary condition in (2.12). They alsy satisfy (2.11) which is equivalent to the curl free equation in (2.12) and completes the existence part of the theorem. The contraction arguments in the above proof automatically provide the uniqueness of the solution.

The stability estimate (2.13) comes from the integral equation (2.14). Let's consider another solution of a perturbed problem,

$$
\begin{aligned}
& \tilde{r}^{11}(x, y)=\tilde{b}^{1}\left(x, y_{0}(x)\right)+\int_{y_{0}(x)}^{y} \tilde{a}^{11} \tilde{r}^{11}(x, t)+\tilde{a}^{12} \tilde{r}^{22}(x, t) d t, \\
& \tilde{r}^{22}(x, y)=\tilde{b}^{2}\left(x_{0}(y), y\right)+\int_{x_{0}(y)}^{x} \tilde{a}^{21} \tilde{r}^{11}(t, y)+\tilde{a}^{22} \tilde{r}^{22}(t, y) d t .
\end{aligned}
$$

Then, the difference is estimated by

$$
\begin{aligned}
& \left\|r^{11}-\tilde{r}^{11}\right\|_{\infty}+\left\|r^{22}-\tilde{r}^{22}\right\|_{\infty} \leq\left\|b^{1}-\tilde{b}^{1}\right\|_{\infty}+\left\|b^{2}-\tilde{b}^{2}\right\|_{\infty} \\
& \quad+2 D\left(\max \left\|a^{i j}-\tilde{a}^{i j}\right\|_{\infty}\left(\left\|b^{1}\right\|_{\infty}+\left\|b^{2}\right\|_{\infty}\right)+\max \left\|\tilde{a}^{i j}\right\|_{\infty}\left(\left\|b^{1}-\tilde{b}^{1}\right\|_{\infty}+\left\|b^{2}-\tilde{b}^{2}\right\|_{\infty}\right)\right),
\end{aligned}
$$

where $D>0$ is the diameter of the domain $\Omega$. The coefficients $a^{i j}$ and $\tilde{a}^{i j}$ are decided by $\mathbf{F}_{k}, \nabla \mathbf{F}_{k}, \tilde{\mathbf{F}}_{k}$ and $\nabla \tilde{\mathbf{F}}_{k}$ through the relation (2.11). Therefore, the relation can be written in the form of (2.13) with the coefficient $C$ that depends on $\Omega, \mathbf{F}_{k}, \nabla \mathbf{F}_{k}, \tilde{\mathbf{F}}_{k}, \nabla \tilde{\mathbf{F}}_{k}, b^{k}$ and $\tilde{b}^{k}$ with $k=1,2$.

Remark 2.6 (Existence). The existence part of this kind of inverse problem has been mostly overlooked since current density data are assumed to be generated using an existing conductivity body. However, if a noise has been added to current data, then the existence should be the first concern. In particular, one should figure out the right amount of data to avoid an over determined problem. Theorem 2.5 shows that two sets of internal current density and boundary values on $\Gamma_{i}^{-}$are the correct amount of data that allow the existence of an orthotropic conductivity.

\section{VRN FOR ORTHOTROPIC CONDUCTIVITY RECONSTRUCTION}

We now construct an orthotropic conductivity reconstruction algorithm using a rectangular virtual resistive network system. The register of a horizontal edge approximates the eigenvalue $r^{11}$ of the orthotropic conductivity and the register of a vertical edge approximates the other eigenvalue $r^{22}$. For a presentational simplicity we consider a rectangular domain $\Omega=(0,1) \times$ $(0,1)$. Then, the boundary components defined in $(2.9)$ are given as $\Gamma_{1}^{0}=\{0\} \times[0,1] \cup\{1\} \times[0,1]$, $\Gamma_{1}^{-}=(0,1) \times\{0\}$ and $\Gamma_{1}^{+}=(0,1) \times\{1\}$. Similarly, the boundary components defined in $(2.10)$ are $\Gamma_{2}^{0}=[0,1] \times\{0\} \cup[0,1] \times\{1\}, \Gamma_{2}^{-}=\{0\} \times(0,1)$ and $\Gamma_{2}^{+}=\{1\} \times(0,1)$.

3.1. Mimetic discretization. We discretize the domain into $n \times n$ smaller squares. Then, the mesh points $\left(x^{i}, y^{j}\right)$ are with $0 \leq i, j \leq n$. Hence there are $(n+1)^{2}$ vertices, $n(n+1)$ horizontal edges, $n(n+1)$ vertical edges, and $n^{2}$ square cells. In a mimetic approach the potential $u$ is assigned at these vertices, $u^{i j} \cong u\left(x^{i}, y^{j}\right)$, and the value of the steam function $\psi$ is assigned at the center of each cell, $\psi_{i+\frac{1}{2}} j+\frac{1}{2} \cong \psi\left(x^{i+1 / 2}, y^{j+1 / 2}\right)$. The current density $\mathbf{F}$ and the electrical field $\mathbf{E}$ are assigned along edges. For example, $F^{i j+\frac{1}{2}}$ is the component of the current density $\mathbf{F}$ in the direction connecting two mesh points $\left(x^{i}, y^{j}\right)$ and $\left(x^{i}, y^{j+1}\right)$. Notice that $\left(x^{i}, y^{j+\frac{1}{2}}\right)$ denotes the midpoint of the this edge. Using the relation $\mathbf{E}=-\nabla u$ and $\mathbf{F}=\nabla^{\perp} \psi$, we obtain

$$
\begin{aligned}
E^{i+\frac{1}{2} j} & =-\frac{u^{i+1 j}-u^{i j}}{x^{i+1}-x^{i}}, & E^{i j+\frac{1}{2}} & =-\frac{u^{i j+1}-u^{i j}}{y^{j+1}-y^{j}}, \\
F^{i+\frac{1}{2} j} & =\frac{\psi_{i+\frac{1}{2} j+\frac{1}{2}}-\psi_{i+\frac{1}{2} j-\frac{1}{2}}}{y^{j+\frac{1}{2}}-y^{j-\frac{1}{2}}}, & F^{i j+\frac{1}{2}} & =-\frac{\psi_{i+\frac{1}{2} j+\frac{1}{2}}-\psi_{i-\frac{1}{2} j+\frac{1}{2}}}{x^{i+\frac{1}{2}}-x^{i-\frac{1}{2}}} .
\end{aligned}
$$

The conductivity and the resistivity are related to $\mathbf{F}$ and $\mathbf{E}$ by Ohm's law. Therefore, it is natural to assign conductivity and resistivity along the edges such as $\sigma^{i+\frac{1}{2} j}$ and $r^{i+\frac{1}{2} j}$. In the 


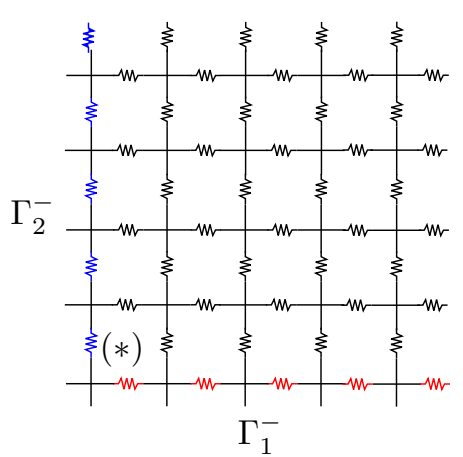

(a) rectangular VRN

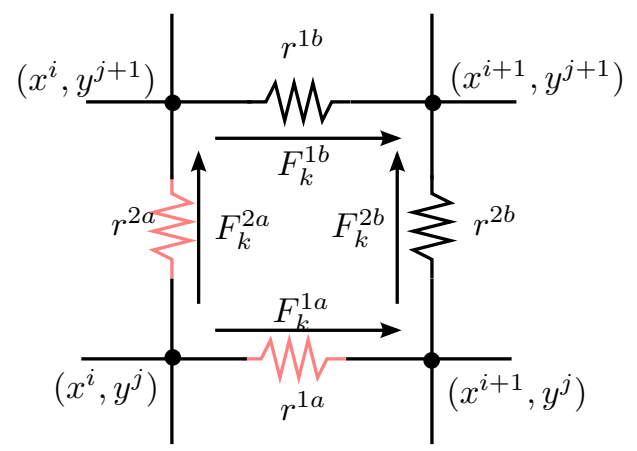

(b) components of a cell

FiguRE 3. A rectangular network. If the resistivity values of boundary resistors along two sides of the rectangular domain, the colored (or grey) ones, are given, the others can be computed by a cell by cell local computation.

system there are $n^{2}+n$ vertical edges and the same number of horizontal edges. The boundary condition $r^{11}=b^{1}$ on the boundary $\Gamma_{1}^{-}$decides the values along $n$ bottom edges and the other boundary condition $r^{22}=b^{2}$ on the boundary $\Gamma_{2}^{-}$decides the values along $n$ left side edges. Therefore, $2 n$ number of edges are decided and we are supposed to decide the other $2 n^{2}$ edges.

Let $D$ be the domain of a cell in Figure 3(b). Then, the integration of (2.12) over the area $D$ gives

$$
0=\int_{D} \nabla \times\left(\boldsymbol{r} \mathbf{F}_{k}\right) d x=\oint_{\partial D} \boldsymbol{r} \mathbf{F}_{k}(z) \cdot d z
$$

We assume that each edge has the same length and hence the resistor and resistivity are equivalent. Then, line integrations of the above equations for $k=1$ and 2 give

$$
\begin{aligned}
& r^{1 a} F_{1}^{1 a}+r^{2 b} F_{1}^{2 b}-r^{1 b} F_{1}^{1 b}-r^{2 a} F_{1}^{2 a}=0, \\
& r^{1 a} F_{2}^{1 a}+r^{2 b} F_{2}^{2 b}-r^{1 b} F_{2}^{1 b}-r^{2 a} F_{2}^{2 a}=0 .
\end{aligned}
$$

Note that there are $n^{2}$ number of cells and hence there are $n^{2}$ equations for each set of current density $\mathbf{F}$. Therefore, two sets of current data, $\mathbf{F}_{1}$ and $\mathbf{F}_{2}$, may decide the resistivity of $2 n^{2}$ edges. Suppose that $r^{1 a}$ and $r^{2 a}$ are obtained from the boundary condition or from previous steps. Then, we have two unknowns and two equations. If we return to the mimetic notation, this system is written as

$$
\left(\begin{array}{ll}
F_{1}^{i+\frac{1}{2} j+1} & -F_{1}^{i+1 j+\frac{1}{2}} \\
F_{2}^{i+\frac{1}{2} j+1} & -F_{2}^{i+1 j+\frac{1}{2}}
\end{array}\right)\left(\begin{array}{l}
r^{i+\frac{1}{2} j+1} \\
r^{i+1 j+\frac{1}{2}}
\end{array}\right)=\left(\begin{array}{ll}
F_{1}^{i+\frac{1}{2} j} & -F_{1}^{i j+\frac{1}{2}} \\
F_{2}^{i+\frac{1}{2} j} & -F_{2}^{i j+\frac{1}{2}}
\end{array}\right)\left(\begin{array}{l}
r^{i+\frac{1}{2} j} \\
r^{i j+\frac{1}{2}}
\end{array}\right),
$$

where $0 \leq i, j<n$ and $r^{i+\frac{1}{2}} 0$ and $r^{0 j+\frac{1}{2}}$ are given from the boundary conditions on $\Gamma_{1}^{-}$and $\Gamma_{2}^{-}$, respectively. Notice that the determinant of the coefficient matrixes is the network version of $\mathbf{F}_{1} \times\left(-\mathbf{F}_{2}\right)$, which has been assumed not to be zero if the pair $\mathbf{F}_{1}$ and $\mathbf{F}_{2}$ are admissible. Therefore, we may obtain the unknown resistors by taking the inverse matrix. If we return to the mimetic notation, it is written as

$$
\left(\begin{array}{l}
r^{i+\frac{1}{2} j+1} \\
r^{i+1 j+\frac{1}{2}}
\end{array}\right)=A\left(\begin{array}{l}
r^{i+\frac{1}{2} j} \\
r^{i j+\frac{1}{2}}
\end{array}\right), \quad A:=\left(\begin{array}{ll}
F_{1}^{i+\frac{1}{2} j+1} & -F_{1}^{i+1 j+\frac{1}{2}} \\
F_{2}^{i+\frac{1}{2} j+1} & -F_{2}^{i+1 j+\frac{1}{2}}
\end{array}\right)^{-1}\left(\begin{array}{ll}
F_{1}^{i+\frac{1}{2} j} & -F_{1}^{i j+\frac{1}{2}} \\
F_{2}^{i+\frac{1}{2} j} & -F_{2}^{i j+\frac{1}{2}}
\end{array}\right) .
$$


3.2. Domains of dependence and influence. In this section we study how a noise propagates when the algorithm (3.4) is used. This information is useful when we design a different network system in the following section. From the relation (3.4) we can easily see that the resistors in the domain $x<x^{i+1}$ and $y<y^{j+1}$ are needed to compute the horizontal resistor at the point $\left(x^{i+\frac{1}{2}}, y^{j+1}\right)$ and vertical resistor at $\left(x^{i+1}, y^{j+\frac{1}{2}}\right)$. Therefore, we may say that the domain of dependence for the resistivity at $\left(x_{0}, y_{0}\right) \in \Omega$ is all points $(x, y) \in \Omega$ such that $x<x_{0}$ and $y<y_{0}$. Similarly, the domain of influence of the information at the point $\left(x_{0}, y_{0}\right)$ is for $x>x_{0}$ and $y>y_{0}$. However, this is true only partly. The two coefficient matrixes (3.4) are almost identical to each other if the data are smooth and the mesh size is small. Therefore, the matrix $A$ in (3.4) is almost the identity matrix, but with a small perturbation. The resistivity of a horizontal edge, $r^{i+\frac{1}{2} j+1}$, is mostly decided by $r^{i+\frac{1}{2} j}$ and, inductively, by the information along the edges with $\left(x^{i+\frac{1}{2}}, y^{k}\right)$ for $k \leq j$. Therefore, the noise of the eigenvalue $r^{11}$ along a vertical column of horizontal edges stays inside the column. In Figure 9(a) a reconstructed image for the horizonal edges is given and one may observe such vertical lines of noises. Similarly, a vertical resistor mostly depends on the ones in the horizontal row and one may observe noisy horizontal lines in Figure 9(d).

One may observe this phenomenon directly from the differential equation. The system of two equations for the orthotropic problem also is hyperbolic. They are

$$
\begin{aligned}
& \nabla \times\left(\boldsymbol{r} \mathbf{F}_{1}\right)=0, \\
& \nabla \times\left(\boldsymbol{r} \mathbf{F}_{2}\right)=0,
\end{aligned}
$$

where $\boldsymbol{r}=r^{i j} \delta^{i j}$ is diagonal matrix field. The system is written as

$$
\left(\begin{array}{l}
\partial_{y} r^{11} \\
\partial_{x} r^{22}
\end{array}\right)=\left(\begin{array}{ll}
-F_{1}^{x} & F_{1}^{y} \\
-F_{2}^{x} & F_{2}^{y}
\end{array}\right)^{-1}\left(\begin{array}{ll}
\partial_{y} F_{1}^{x} & -\partial_{x} F_{1}^{y} \\
\partial_{y} F_{2}^{x} & -\partial_{x} F_{2}^{y}
\end{array}\right)\left(\begin{array}{l}
r^{11} \\
r^{22}
\end{array}\right)=: \mathbf{A}\left(\begin{array}{l}
r^{11} \\
r^{22}
\end{array}\right)
$$

The system contains partial derivatives of the horizontal resistivity $r^{11}$ with respect to $y$ variable only. Hence, the characteristic lines of $r^{11}$ are vertical ones. Hence, if the boundary function $b^{1}$ is given on $\Gamma_{1}^{-}$, which is on the $x$-axis, then $r^{11}$ is computed by integrating the first equation along the characteristic lines which are vertical. The derivative of the vertical resistivity $r^{22}$ is with respect to $y$ variable only. Hence, similarly, vertical lines are characteristic lines of $r^{22}$ and, since the boundary function $b^{2}$ is given on $\Gamma_{2}^{-}$, which is on the $y$-axis, then $r^{22}$ is computed by integrating the second equation along horizontal lines.

3.3. VRN of layered bricks. If the network system is aligned along characteristic lines, the noise effect is not cancelled each other, but stays inside the characteristic lines. As its consequence, the recovered image contains lots of stripes along the characteristic lines and the recovered image shows a poor resolution. Such a phenomenon has been observed from most of algorithms for isotropic conductivity reconstruction (see [17]). One may observe a more serious situation if the conductivity is not isotropic. In this section we introduce a network system to avoid such a noise propagation phenomenon.

One might consider a network system that cells are tilted by 45 degree as in Figure 4(a). Then the cells are aligned in a direction that intersect the characteristic lines with 45 degree angle. In this way, we may expect to neutralize the effect of noise. However, this network does not work and we cannot recover the eigenvalues ${ }^{1}$. The main idea of rectangular network system for an orthotropic conductivity reconstruction is that the horizontal and vertical resistors may represent the eigenvalues $r^{11}$ and $r^{22}$, respectively. However, as soon as we use tilted edges, we loose this advantage.

\footnotetext{
${ }^{1}$ Suppose that $r^{11} \neq r^{22}$ and they are constants. Then the resistivity along the tilted edges of the network in Figure 4(a) are identical. In other words, this network does not recognize the orthotropic structure.
} 


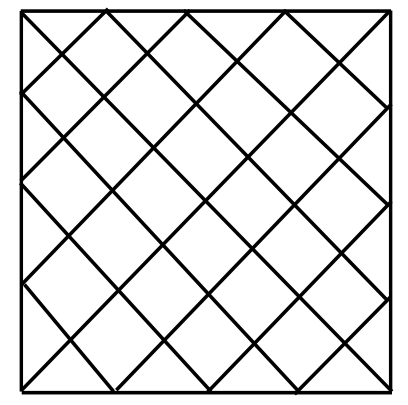

(a) diagonal arrangement

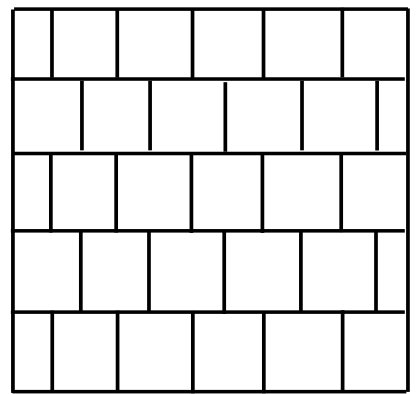

(b) layered bricks

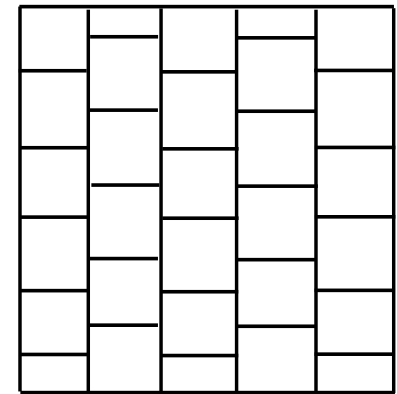

(c) rotated layered bricks

FiguRE 4. The VRN given in (a) fails. Layered bricks in (b) gives stable images of $r^{11}$ and the ones in (c) gives stable images of $r^{22}$.

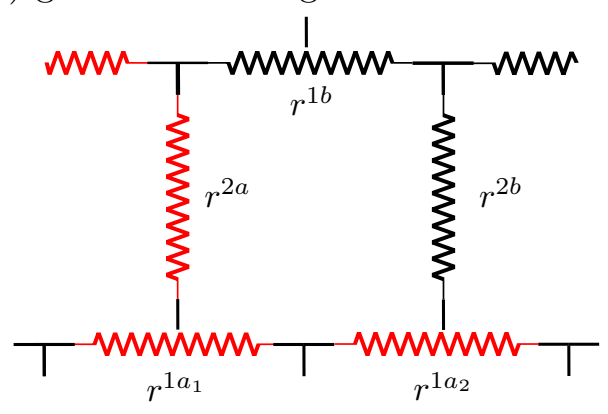

FiguRe 5. Structure of a layered brick. One side of each cell consists of two half edges of neighboring cells.

The network system we are going to use is of layered bricks type given in Figure 4(b). In this network, all edges are in horizontal or vertical directions and they will be used to recover the eigenvalues in the horizontal or vertical directions, respectively. The cells are aligned horizontally and hence horizonal stripes are not avoidable. However, the vertical stripes can be disappeared. An example of a recovered horizontal component $r^{11}$ is given in Figure 8(b) using this layered bricks type VRN. The vertical stripes observed in Figure 9(a) have been disappeared. However, horizontal stripes are observed from a recovered resistivity $r^{22}$ in Figure 8(e). To obtain an improved image for the resistivity $r^{22}$ we consider a rotated layered bricks given in Figure $8(\mathrm{c})$. In this VRN system the cells are aligned vertically and hence the reconstructed image for $r^{11}$ may contain noise vertical stripes as in Figure 8(c). However, the horizontal stripes of the reconstructed image for $r^{22}$ may disappear as in Figure 8(f). The VRN system we choose to reconstruct the orthotropic conductivity is the one of layered bricks. In computations the resistivity images are constructed twice using the layered bricks and the rotated ones and the image for horizontal component $r^{11}$ is taken from the layered bricks VRN and the ones for vertical component $r^{22}$ is taken from the rotated bricks VRN. The other two images with stripes are forgotten.

The algorithm for the VRN of layered bricks is similar to the one for the rectangular ones. Consider the cell given in Figure 5. Suppose that the resistors $r^{1 a_{1}}, r^{1 a_{2}}$ and $r^{2 a}$ are given by the previous steps or by the boundary condition. We want to decide the other resistors $r^{2 b}$ and $r^{1 b}$. The idea is to replace the $r^{1 a}$ in (3.3) with

$$
r^{1 a}:=\frac{1}{2}\left(r^{1 a_{1}}+r^{1 a_{2}}\right)
$$


Then, we obtain an algorithm for the layered bricks:

$$
\left(\begin{array}{l}
r^{i+\frac{1}{2} j+1} \\
r^{i+1 j+\frac{1}{2}}
\end{array}\right)=B\left(\begin{array}{c}
\frac{1}{2} r^{i j} \\
\frac{1}{2} r^{i+1 j} \\
r^{i j+\frac{1}{2}}
\end{array}\right)
$$

where $B$ is a $2 \times 3$ matrix given by

$$
B:=\left(\begin{array}{ll}
F_{1}^{i+\frac{1}{2} j+1} & -F_{1}^{i+1 j+\frac{1}{2}} \\
F_{2}^{i+\frac{1}{2} j+1} & -F_{2}^{i+1 j+\frac{1}{2}}
\end{array}\right)^{-1}\left(\begin{array}{lll}
F_{1}^{i j} & F_{1}^{i+1 j} & -F_{1}^{i j+\frac{1}{2}} \\
F_{2}^{i j} & F_{2}^{i+1 j} & -F_{2}^{i j+\frac{1}{2}}
\end{array}\right) .
$$

One may similarly obtain an algorithm for the rotated layered bricks which is omitted here.

\section{Numerical Simulations}

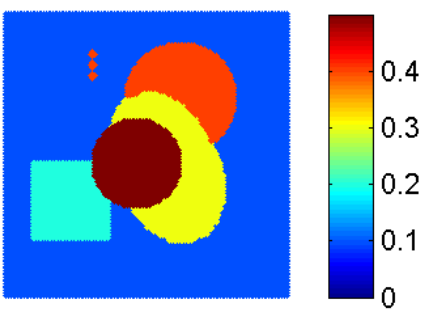

(a) $r^{11}$ : horizontal direction

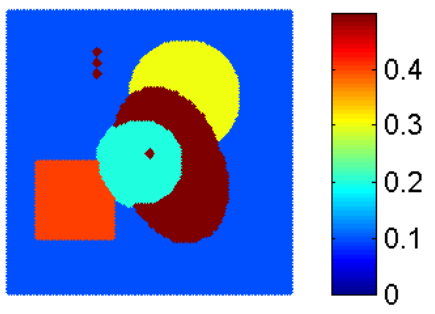

(b) $r^{22}$ : vertical direction

FiguRE 6. Target images of horizontal and vertical eigenvalues that are used in simulations of orthotropic resistivity reconstruction and simulation setup.

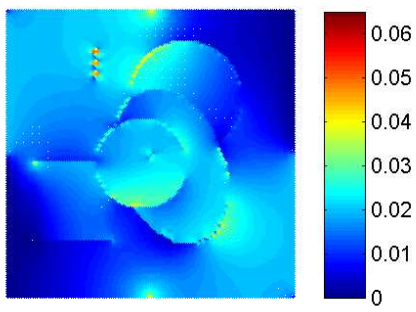

(a) $x$ component of $-\mathbf{J}_{1}$

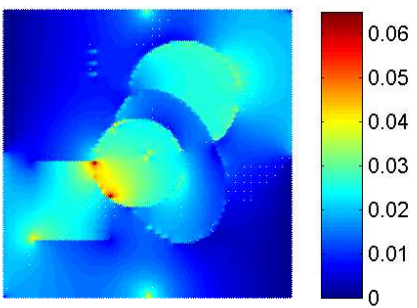

(c) $x$ component of $\mathbf{J}_{2}$

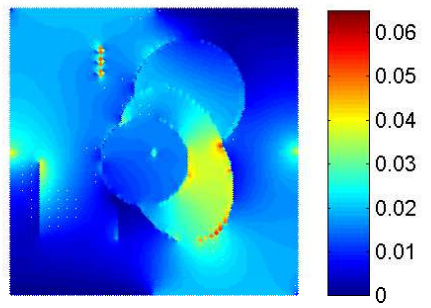

(b) $y$ component of $\mathbf{J}_{1}$

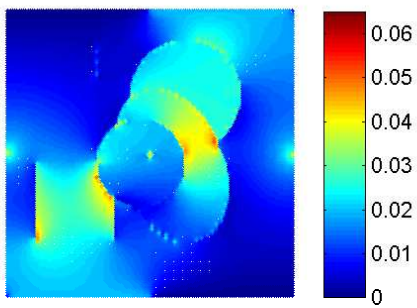

(d) $y$ component of $\mathbf{J}_{2}$

Figure 7. Two current data $\mathbf{J}_{1}$ and $\mathbf{J}_{2}$ used in the simulations. 
4.1. Target conductivity and adding noises. The target conductivity images for the numerical simulations in this section are given in Figure 6. The orthotropic conductivity $\boldsymbol{\sigma}=\left(\sigma^{i j} \delta^{i j}\right)$ in two space dimensions consists of two components of eigenvalues $\sigma^{11}$ and $\sigma^{22}$. The resistivity is simply its inverse matrix $\boldsymbol{r}=\left(r^{i j}\right)=\boldsymbol{\sigma}^{-1}$. Therefore, $r^{12}=r^{21}=0$ and $r^{11}=1 / \sigma^{11}$ and $r^{22}=1 / \sigma^{22}$. In numerical computations we find the resistivity using VRN and display the images in terms of conductivity since conductivity images are commonly used in EIT and MREIT. The eigenvalues are scaled as

$$
0.1 \leq \sigma^{11}, \sigma^{22} \leq 0.5, \quad \text { or equivalently, } \quad 2 \leq r^{11}, r^{22} \leq 10 .
$$

The color bars are given with images in Figure 6. We use the same color bar in the reconstructed conductivity images and do not display the color bar any more.

The domain of the conductivity body used in the simulation is of a square shape with the physical size of $50 \mathrm{~cm} \times 50 \mathrm{~cm}$. We used two sets of electrical currents. To obtain the first one an electrical current of total amount $10 \mathrm{~mA}$ was injected uniformly through the boundary from the middle of bottom to the middle of the right side. The same amount was extracted uniformly through the boundary from the middle of the left side to the middle of the top. The current density obtained by solving the forward problem (2.6) using this boundary current density is denoted $\mathbf{J}_{1}$ and given in Figures $7(\mathrm{a})$ and $7(\mathrm{~b})$. The same amount current was injected and extracted from the other parts of the boundary and is called $\mathbf{J}_{2}$. The corresponding images are given in Figures 7(c) and 7(d). The locations of injections and extractions are chosen so that the main directions of the currents are perpendicular to each other and hence the chance to have $\mathbf{J}_{1} \times \mathbf{J}_{2}=0$ is minimized. The sizes of absolute value of data were

$$
\begin{aligned}
& 3.7 \times 10^{-5}\left[A / m^{2}\right] \leq\left|\mathbf{J}_{1}\right| \leq 5.3 \times 10^{-2}\left[A / m^{2}\right], \\
& 1.2 \times 10^{-4}\left[A / m^{2}\right] \leq\left|\mathbf{J}_{2}\right| \leq 6.2 \times 10^{-2}\left[A / m^{2}\right] .
\end{aligned}
$$

Remember that the current density $\mathbf{J}_{1}$ and $\mathbf{J}_{2}$ are obtained from the rectangular VRN introduced in Figure 3, but not from the layered bricks. Therefore, $\mathbf{J}_{1}$ and $\mathbf{J}_{2}$ are not exact current densities with respect to the layered bricks. For example, consider the reconstructed conductivity images given in Figure 8. One can see that the rectangular VRN reconstructs the target conductivity images exactly using these current densities. However, the reconstruct images obtained by layered bricks VRN and a rotated one contain noisy stripes.

We add a noise to the current data in the following simulations to test the stability of algorithms. We add two kinds of noises, which will be called multiplicative and additive noises. The multiplicative noise of $p \%$ is a random noise computed by

$$
\left(\frac{p}{100}|\mathbf{J}(\mathbf{x})|\right) X
$$

where $-1 \leq X \leq 1$ is the random variable with a uniform distribution. The size of the multiplicative noise is proportional to the size of current $\mathbf{J}$.

The additive noise follows a normal distribution of average $\mu=0$ and a standard deviation s.d. $>0$, say $N(\mu=0, s . d$.). (Note that the standard deviation is usually denoted by $\sigma$, but we have already used it for conductivity.) Summing them up gives the current density data used in the simulation:

$$
\mathbf{F}=\mathbf{J}+\left(\frac{p}{100}|\mathbf{J}|\right) X+N(0, \text { s.d. }) .
$$

As a measure of additive noise we consider the ratio of signal to standard deviation,

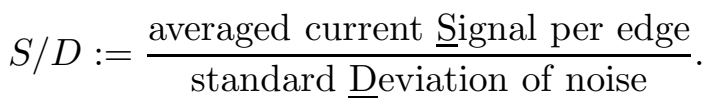

There are 23, 863 network edges in the interior circular domain and the average current used in the simulation is about $0.078 \mathrm{~mA}$. For example, in the simulation of Figure 12(d) an additive 


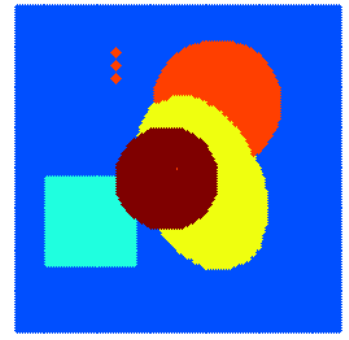

(a) $r^{11}$ (rectangular $\left.\mathrm{VRN}\right)$

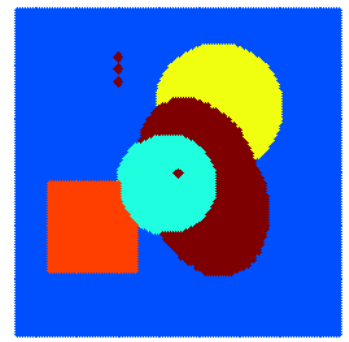

(d) $r^{22}$ (rectangular VRN)

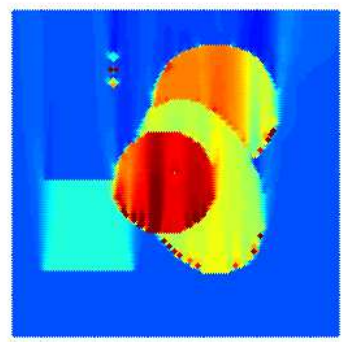

(b) $r^{11}$ (layered bricks VRN)

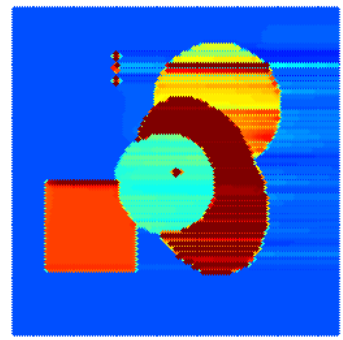

(e) $r^{22}$ (layered bricks VRN)

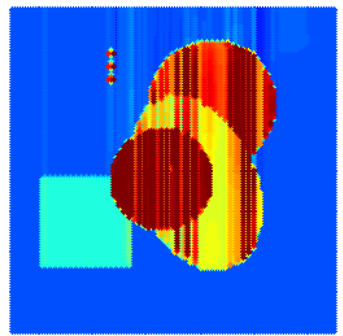

(c) $r^{11}$ (rotated bricks VRN)

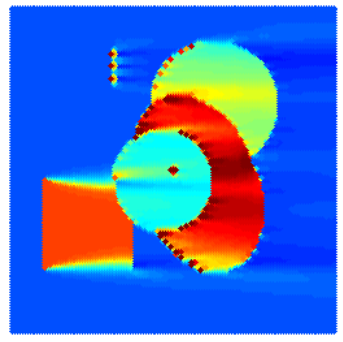

(f) $r^{22}$ (rotated bricks VRN)

FIgURE 8. Reconstructed orthotropic conductivity images using current densities generated by rectangular VRN without extra noises added.

noise with a standard deviation s.d. $=0.0026 \mathrm{~mA}$ has been added to each of the edges and hence the ratio is $S / D=30$.

4.2. Rectangular cells versus layered bricks. Now we are ready to present the numerical properties of VRN method for the reconstruction of orthotropic conductivity. We first compare the properties of using rectangular cells given in Figure 3 and of using the layered bricks given in Figures 4(b), 4(c) and 5. In Figure 8 recovered conductivity images are given using the three different VRN systems without adding any extra noise. First observe that the reconstructed images with the rectangular cells, Figures 8(a) and 8(d), are the exact target conductivity. This is not surprising since the current densities $\mathbf{J}_{1}$ and $\mathbf{J}_{2}$ have been generated using this rectangular VRN system. This computation result simply indicates that the numerical process is reversible, which is a nice property of the mimetic discretization.

Other four figures are obtained using layered bricks and rotated layered bricks. These images contain noise effects, which is natural since the current density is not the one obtained from these VRN. Remember that the characteristic lines for the horizontal eigenvalue $r^{11}$ are vertical lines. Such a vertical noise lines are observed from the rotated layered bricks in Figure 8(c) and a similar phenomenon is observed in Figure 8(b) which is obtained using layered bricks. The difference is that the vertical noise lines from layered bricks are blurred in compare with the one of rotated ones. Similar phenomenon is observed from the reconstructed images of the vertical eigenvalue $r^{22}$. In this case the noise lines from the rotated layered bricks are blurred.

For a fair comparison of these methods a multiplicative noise of $10 \%$ is added to the current densities $\mathbf{J}_{1}$ and $\mathbf{J}_{2}$. The reconstructed images for the eigenvalue $r^{11}$ in the horizontal direction are given in the first row of Figure 9. The noisy vertical stripes are very strong in Figures 9(a) and 9(c). However, many of the stripes Figure 8(b) are disappeared. This is the one obtained by the layered bricks. The images for the vertical eigenvalue $r^{22}$ are given in the second row of Figure 9. In this case horizontal stripes are found in Figures 9(d) and 9(e). Many of these 


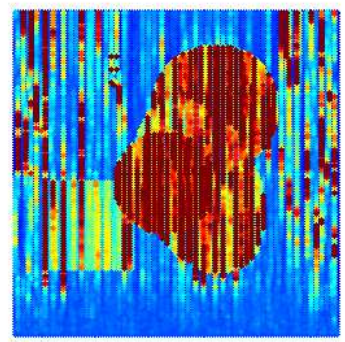

(a) $r^{11}$ (rectangular $\left.\mathrm{VRN}\right)$

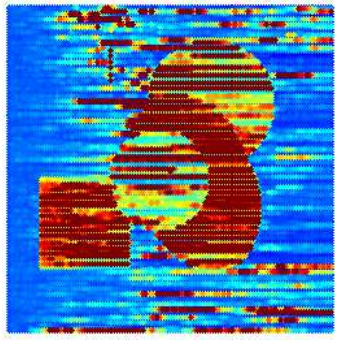

(d) $r^{22}$ (rectangular VRN)

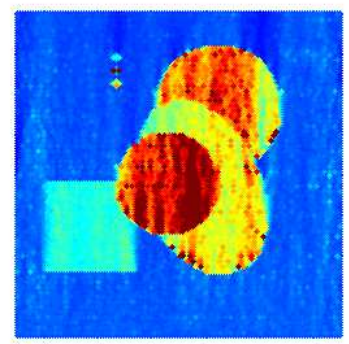

(b) $r^{11}$ (layered bricks VRN)

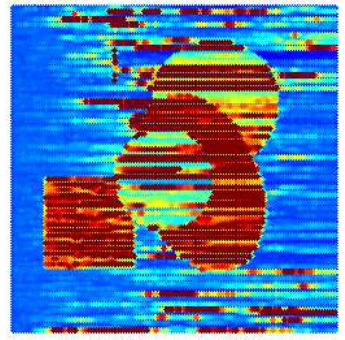

(e) $r^{22}$ (layered bricks VRN)

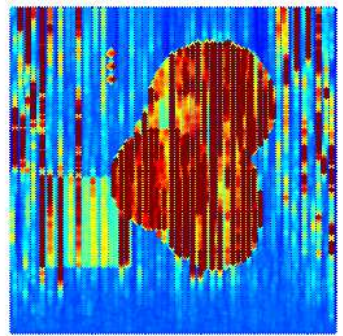

(c) $r^{11}$ (rotated bricks VRN)

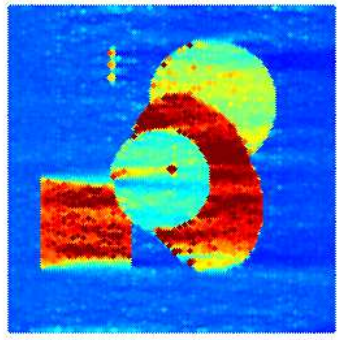

(f) $r^{22}$ (rotated bricks VRN)

FIgURE 9. Reconstructed orthotropic conductivity images using current densities generated by rectangular VRN after adding $10 \%$ multiplicative noise.

stripes are blurred and disappeared in Figure 8(f), which is the one obtained by the rotated layered bricks.

The numerical simulations in this section show the importance of designing a network system in a way that the cells are not aligned in the direction of characteristic lines. We could not design a one that removes both of the vertical and horizontal noise lines and gives acceptable images for both of $r^{11}$ and $r^{22}$. What we did in the numerical simulations is to compute the four images using layered bricks and its rotated ones and pick the image for $r^{11}$ from layered bricks as in Figure 9(b) and the image for $r^{22}$ from rotated layered bricks as in Figure 9(f). We call this method layered bricks VRN in the following sections.

4.3. Regularization. In this section we introduce the regularization technique used in this paper. If a noise is included in the current data $\mathbf{F}_{1}$ and $\mathbf{F}_{2}$, then this regularization process may help us to improve the quality of reconstructed conductivity images. The regularization process is basically a smoothing process that reduces the size of a noise by convoluting the data with a kernel matrix of weight coefficients. We use a typical kernel matrix,

$$
\left(\begin{array}{lll}
0.0251 & 0.1453 & 0.0251 \\
0.1453 & 0.3183 & 0.1453 \\
0.0251 & 0.1453 & 0.0251
\end{array}\right),
$$

which gives the weights of 9 nearby cells for the averaging process. Notice that some of the 9 cells are missing when the smoothing process is performed at a boundary cell. One of the simplest ways to handle the situation is to use the value at the closest cell for a missing cell value, which is the way we did in the simulation.

Reconstructed images are given in Figure 10 to compare the effect of the regularization process. These conductivity images are obtained using rectangular and layered bricks VRNs after adding 10\% multiplicative noise to the current densities. The images in the first row 


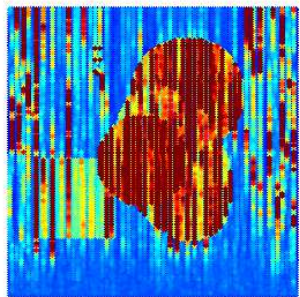

(a) $r^{11}$ (rectangular)

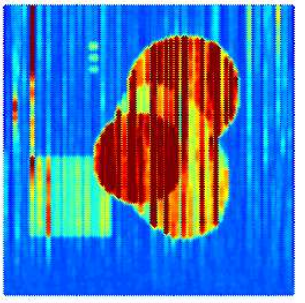

(e) $r^{11}$ (rectangular)

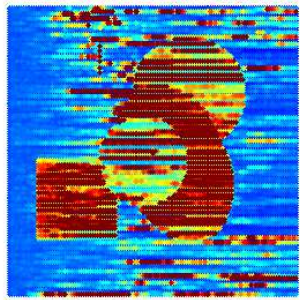

(b) $r^{22}$ (rectangular)

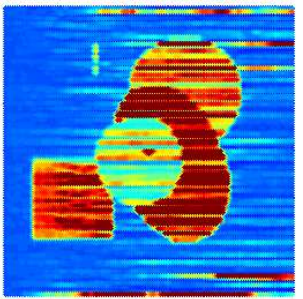

(f) $r^{22}$ (rectangular)

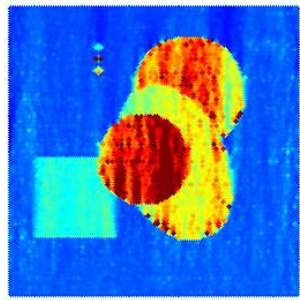

(c) $r^{11}$ (layered bricks)

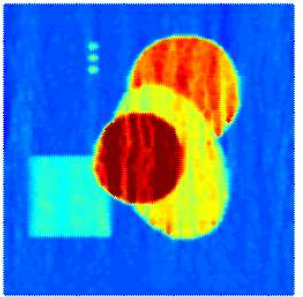

(g) $r^{11}$ (layered bricks)

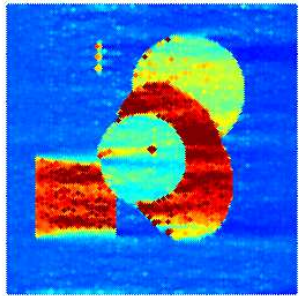

(d) $r^{22}$ (layered bricks)

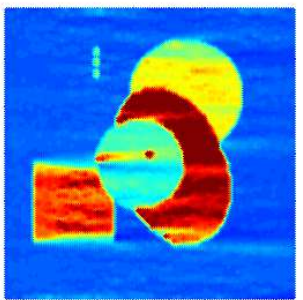

(h) $r^{22}$ (layered bricks)

FIGURE 10. Reconstructed orthotropic conductivity images with $10 \%$ multiplicative noise. The first row is obtained without any regularization process and the second row is with the smoothing process.

are obtained without any regularization process, which are the same ones in Figure 9. These images are given here again for an easier comparison. The second row is obtained after the smoothing process using the kernel in (4.3). The images obtained from rectangular VRN have been improved considerably. The noise stripes became thinner after smoothing the noised data. As a result the boundaries of the inside structures are clearer. Furthermore, the small three dots in the upper left quarter of the domain can be clearly observed after regularization process. However, this improvement is not as big as the isotropic case in [17]. We can similarly observe an improvement of the recovered images when the layered bricks VRN is used. The stripes became more smeared and the conductivity values in the interior structures are closer to the target conductivity in Figure 6. It seems that the smoothing effect is already included in the layered bricks and the improvement is not as substantial as the rectangular VRN case. However, the images recovered by layered bricks VRN are more stable and closer to the target conductivity than the ones by rectangular VRN.

Remark 4.1. Observe that there are singularities in current density data, Figures 7, at the corners of the domain. These singularities reflect the singularities of the domain and are required to reconstruct the correct conductivity as in Figure 8(a) and 8(c). Therefore, if the smoothing process removes such singularities, it may give us a trouble. Similarly, observe singularities at the middle of boundaries. These singularities are related to the boundary value of the current, where the middle of the edges are the boundary points of the supports of the boundary values.

4.4. Numerical stability tests. In this section we test the stability of the orthotropic conductivity reconstruction method suggested in this paper using the layered bricks VRN. We increase the noise level as high as possible and test the stability of the reconstruction method . In Figure 11 conductivity images are reconstructed after adding multiplicative noises. The noise level was increased up to $40 \%$. As the noise level increases the noise stripes appear and become thicker. It seems that the noise sensitivity of this example is about two times bigger than the isotropic case in [17]. 


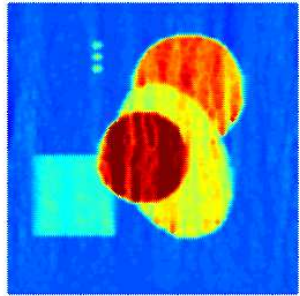

(a) $r^{11}$ with $10 \%$ noise

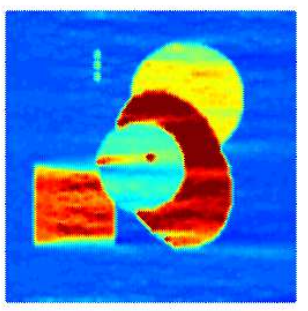

(e) $r^{22}$ with $10 \%$ noise

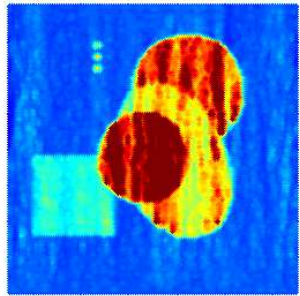

(b) $r^{11}$ with $20 \%$ noise

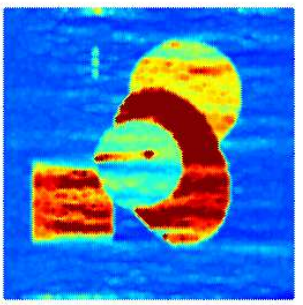

(f) $r^{22}$ with $20 \%$ noise

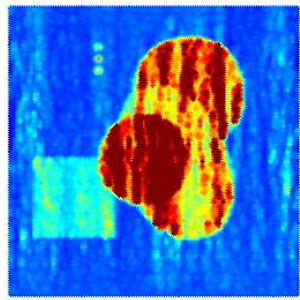

(c) $r^{11}$ with $30 \%$ noise

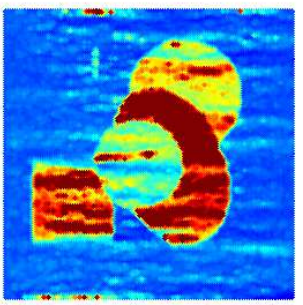

(g) $r^{22}$ with $30 \%$ noise

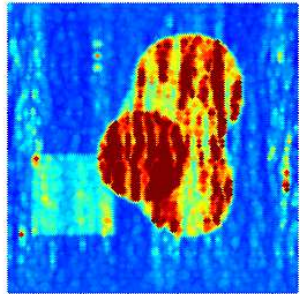

(d) $r^{11}$ with $40 \%$ noise

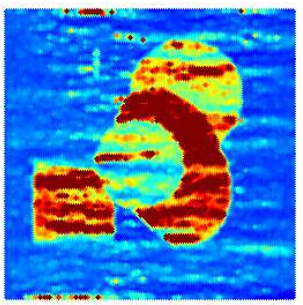

(h) $r^{22}$ with $40 \%$ noise

FiguRE 11. Reconstructed images using layered bricks and regularized data. Multiplicative noises were added to the current data.

In Figure 12 conductivity images are reconstructed after adding additive noises. The noise level was increased up to $S / D=30$. We may observe that the noise effect is severer at the corners of the square domain. These are the regions that the current density is smaller and hence the additive noise is relatively larger in these corners. This phenomenon is not observed when a multiplicative noise is added, which is proportional to the signal size.

One may observe that the noise stripes appear more in the region that the conductivity has a discontinuity in the horizontal or vertical direction. For example, the images of the conductivity in the vertical direction, $r^{22}$, have blurred horizontal stripes that start from regions with horizontal conductivity discontinuity, Figures $11(\mathrm{e})-11(\mathrm{~h})$ and Figures $12(\mathrm{e})-12(\mathrm{~h})$. However, vertical discontinuity edges are reconstructed clearly. Similarly, the images for $r^{11}$ have blurred vertical stripes near the regions with vertical discontinuity, Figures 11(a)-11(d) and Figures $12(\mathrm{a})-12(\mathrm{~d})$. Horizontal discontinuity edges are clearer in these images.

\section{REFERENCES}

1. Giovanni Alessandrini, An identification problem for an elliptic equation in two variables, Ann. Mat. Pura Appl. (4) 145 (1986), 265-295. MR 886713 (88g:35193)

2. - Critical points of solutions of elliptic equations in two variables, Annali della Scuola Normale Superiore di Pisa. Classe di Scienze. Serie IV 14 (1987), no. 2, 229-256 (1988).

3. Habib Ammari, An introduction to mathematics of emerging biomedical imaging, Mathématiques \& Applications (Berlin) [Mathematics \& Applications], vol. 62, Springer, Berlin, 2008. MR 2440857 (2010j:44002)

4. Habib Ammari, Yves Capdeboscq, Hyeonbae Kang, and Anastasia Kozhemyak, Mathematical models and reconstruction methods in magneto-acoustic imaging, European J. Appl. Math. 20 (2009), no. 3, 303-317. MR 2511278 (2010f:35428)

5. Habib Ammari and Hyeonbae Kang, Reconstruction of small inhomogeneities from boundary measurements, Lecture Notes in Mathematics, vol. 1846, Springer-Verlag, Berlin, 2004. MR 2168949 (2006k:35295)

6. Guillaume Bal, Chenxi Guo, and François Monard, Imaging of Anisotropic Conductivities from Current Densities in Two Dimensions, SIAM J. Imaging Sci. 7 (2014), no. 4, 2538-2557. MR 3284567

7. __ Inverse anisotropic conductivity from internal current densities, Inverse Problems 30 (2014), no. 2, 025001, 21. MR 3162103

8. Liliana Borcea, Electrical impedance tomography, Inverse Problems 18 (2002), no. 6, R99-R136. MR 1955896 


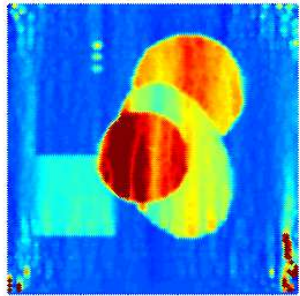

(a) $r^{11}$ with $\mathrm{S} / \mathrm{D}=120$

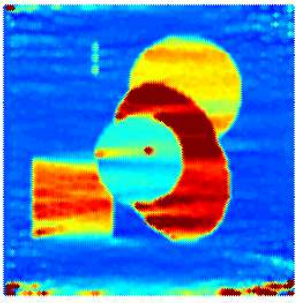

(e) $r^{22}$ with $\mathrm{S} / \mathrm{D}=120$

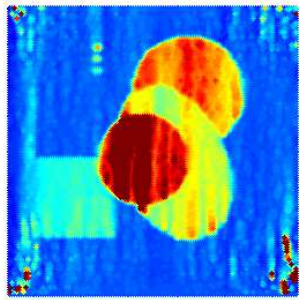

(b) $r^{11}$ with $\mathrm{S} / \mathrm{D}=80$

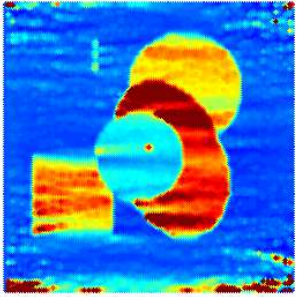

(f) $r^{22}$ with $\mathrm{S} / \mathrm{D}=80$

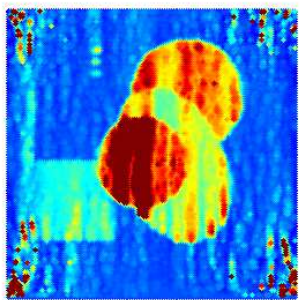

(c) $r^{11}$ with $\mathrm{S} / \mathrm{D}=45$

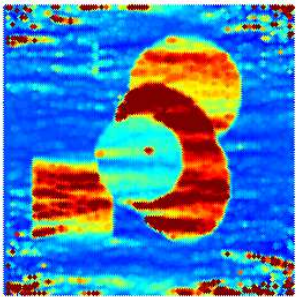

(g) $r^{22}$ with $\mathrm{S} / \mathrm{D}=45$

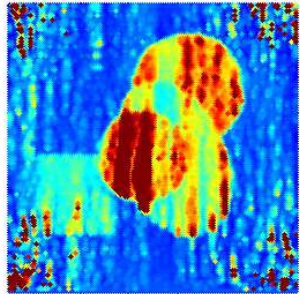

(d) $r^{11}$ with $\mathrm{S} / \mathrm{D}=30$

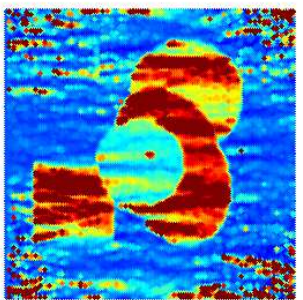

(h) $r^{22}$ with $\mathrm{S} / \mathrm{D}=30$

FiguRE 12. Reconstructed images using layered bricks and regularized data. Additive noises were added to the current data.

9. Nicholas Hoell, Amir Moradifam, and Adrian Nachman, Current density impedance imaging of an anisotropic conductivity in a known conformal class, SIAM J. Math. Anal. 46 (2014), no. 3, 1820-1842. MR 3206987

10. Y.Z. Ider, S. Onart, and W. Lionheart, Uniqueness and reconstruction in magnetic resonance-electrical impedance tomography (MR-EIT), Physiological measurement 24 (2003), 591-604.

11. H.S. Khang, B.I. Lee, S.H. Oh, E.J. Woo, S.Y. Lee, M.H. Cho, Kwon O., Yoon J.R., and J.K. Seo, Jsubstitution algorithm inmagnetic resonance electrical impedance tomography (mreit): phantom experiments for static resistivity images, IEEE Trans. Med. Imaging 21 (2002), 695-702.

12. Y.-J. Kim, O. Kwon, J.K. Seo, and E.J. Woo, Uniqueness and convergence of conductivity image reconstruction in magnetic resonance electrical impedance tomography, Inverse Problems 19 (2003), no. 5, 1213-1225.

13. Ohin Kwon, June-Yub Lee, and Jeong-Rock Yoon, Equipotential line method for magnetic resonance electrical impedance tomography, Inverse Problems 18 (2002), no. 4, 1089-1100.

14. Ohin Kwon, Eung Je Woo, Jeong-Rock Yoon, and Jin Keun Seo, Magnetic resonance electrical impedance tomography (MREIT): simulation study of J-substitution algorithm, Biomedical Engineering, IEEE Transactions on 49 (2002), no. 2, $160-167$.

15. M.G. Lee and Y.-J. Kim, Existence and uniqueness in anisotropic conductivity reconstruction with Faraday's law, preprint (2015).

16. M.G. Lee and Y.-J. Kim, Well-posedness of the conductivity reconstruction from an interior current density in terms of Schauder theory, Quart. Appl. Math. (To appear).

17. M.G. Lee, M.-S. Ko, and Y.-J. Kim, Virtual resistive network and conductivity reconstruction with Faraday's law, Inverse Problems 30 (2014), no. 12, 21pp.

18. François Monard and Guillaume Bal, Inverse anisotropic diffusion from power density measurements in two dimensions, Inverse Problems 28 (2012), no. 8, 084001, 20. MR 2956557

19. Inverse anisotropic conductivity from power densities in dimension $n \geq 3$, Comm. Partial Differential Equations 38 (2013), no. 7, 1183-1207. MR 3169742

20. Adrian Nachman, Alexandru Tamasan, and Alexandre Timonov, Conductivity imaging with a single measurement of boundary and interior data, Inverse Problems 23 (2007), no. 6, 2551-2563.

21. no. 3, 035014, 16. MR 2480184 (2010g:35340)

22. A.I. Nachman, Global uniqueness for a two-dimensional inverse boundary value problem, Annals of Mathematics (1996), 71-96.

23. Gerard R. Richter, An inverse problem for the steady state diffusion equation, SIAM J. Appl. Math. 41 (1981), no. 2, 210-221. MR 628945 (82m:35143) 
24. Numerical identification of a spatially varying diffusion coefficient, Math. Comp. 36 (1981), no. 154, 375-386. MR 606502 (82c:65055)

25. J. Sylvester and G. Uhlmann, A global uniqueness theorem for an inverse boundary value problem, Annals of Mathematics (1987), 153-169.

26. G. Uhlmann, Electrical impedance tomography and Calderon's problem, Inverse Problems 25 (2009), no. 12, 123011 (39pp).

(Min-Gi Lee)

Computer, Electrical and Mathematical Sciences \& Engineering, KAUST

Thuwal, Saudi Arabia

E-mail address: mgleemail@gmail.com

(Min-Su Ko)

Department of Mathematical Sciences, KAIST

DAEJEON, KoreA

E-mail address: minsu.ko@kaist.ac.kr

(Yong-Jung Kim)

Department of Mathematical Sciences, KAIST

DAEJEOn, Korea

E-mail address: yongkim@kaist.edu 